\title{
Determinism and Contingency Shape Metabolic Complementation in an Endosymbiotic Consortium
}

\begin{abstract}
Miguel Ponce-de-Leon ${ }^{1 *}$, Daniel Tamarit ${ }^{2}$, Jorge Calle-Espinosa ${ }^{1}$, Matteo Mori ${ }^{3}$, Amparo Latorre ${ }^{4,5}$, Francisco Montero ${ }^{1}$ and Juli Pereto ${ }^{5,6 *}$
\end{abstract}

'Departamento de Bioquímica y Biología Molecular I, Facultad de Ciencias Químicas, Universidad Complutense de Madrid, Madrid, Spain, ${ }^{2}$ Science for Life Laboratory, Department of Molecular Evolution, Cell and Molecular Biology, Uppsala University, Uppsala, Sweden, ${ }^{3}$ Department of Physics, University of California, San Diego, La Jolla, CA, United States, ${ }^{4}$ Departament de Genètica, Universitat de València, València, Spain, ${ }^{5}$ Institute for Integrative Systems Biology, Universitat de València-CSIC, València, Spain, ${ }^{6}$ Departament de Bioquímica i Biologia Molecular, Universitat de València, València, Spain

Bacterial endosymbionts and their insect hosts establish an intimate metabolic relationship. Bacteria offer a variety of essential nutrients to their hosts, whereas insect cells provide the necessary sources of matter and energy to their tiny metabolic allies.

OPEN ACCESS

Edited by:

Joerg Graf,

University of Connecticut,

United States

Reviewed by:

João Marcelo Pereira Alves, University of São Paulo, Brazil Laila Pamela Partida-Martinez, Center for Research and Advanced Studies of the National Polytechnic Institute (CINVESTAV), Mexico

*Correspondence: Miguel Ponce-de-Leon migponce@ucm.es Juli Pereto pereto@uv.es

Specialty section: This article was submitted to Microbial Symbioses,

a section of the journal

Frontiers in Microbiology

Received: 23 August 2017 Accepted: 06 November 2017 Published: 22 November 2017

Citation:

Ponce-de-Leon M, Tamarit $D$ Calle-Espinosa J, Mori M, Latorre A Montero F and Pereto J (2017) Determinism and Contingency Shape Metabolic Complementation in an

Endosymbiotic Consortium

Front. Microbiol. 8:2290. doi: 10.3389/fmicb.2017.02290
These nutritional complementations sustain themselves on a diversity of metabolite exchanges between the cell host and the reduced yet highly specialized bacterial metabolism - which, for instance, overproduces a small set of essential amino acids and vitamins. A well-known case of metabolic complementation is provided by the cedar aphid Cinara cedri that harbors two co-primary endosymbionts, Buchnera aphidicola BCc and Ca. Serratia symbiotica SCc, and in which some metabolic pathways are partitioned between different partners. Here we present a genome-scale metabolic network (GEM) for the bacterial consortium from the cedar aphid iBSCc. The analysis of this GEM allows us the confirmation of cases of metabolic complementation previously described by genome analysis (i.e., tryptophan and biotin biosynthesis) and the redefinition of an event of metabolic pathway sharing between the two endosymbionts, namely the biosynthesis of tetrahydrofolate. In silico knock-out experiments with iBSCc showed that the consortium metabolism is a highly integrated yet fragile network. We also have explored the evolutionary pathways leading to the emergence of metabolic complementation between reduced metabolisms starting from individual, complete networks. Our results suggest that, during the establishment of metabolic complementation in endosymbionts, adaptive evolution is significant in the case of tryptophan biosynthesis, whereas vitamin production pathways seem to adopt suboptimal solutions.

Keywords: endosymbiotic bacteria, cross-feeding, metabolic evolution, metabolic modeling, stoichiometric analysis

\section{INTRODUCTION}

Species coexisting in a determined environment establish a network of interactions molded by biotic and abiotic factors (Faust and Raes, 2012; Seth and Taga, 2014). From a molecular point of view, such networks can be considered as an entangled circuitry of various metabolisms interconnected by the exchange of compounds. In mutualistic symbioses, where partners 
exchange nutrients or precursors bi-directionally, the nutritional interdependence will lead, in most cases, to a co-evolutionary process. A particular case occurs when the host cells harbor one or more symbionts inside them (i.e., endosymbionts). As a consequence of the adaptation to the intracellular life, the endosymbionts undergo many biochemical and structural changes, with extreme genome reduction by gene loss being the most dramatic one, compared to their closest free-living relatives (Moran, 1996; Moya et al., 2008; Moran and Bennett, 2014; Manzano-Marín and Latorre, 2016). Gene losses in endosymbionts result in the total or partial demolition of metabolic pathways, and thus endosymbionts become auxotroph for a diversity of compounds, such as nucleotides or amino acids.

The description of nutritional interactions between hosts and symbionts (and among symbionts in consortia) usually relies on the concept of "metabolic complementation", for which at least two distinct meanings have been used. First, we can consider the exchange of essential components (e.g., vitamins and amino acids) between host and endosymbiont (Wu et al., 2006; Moya et al., 2008; López-Sánchez et al., 2009; Macdonald et al., 2012; Russell et al., 2013), a phenomenon usually known as cross-feeding. Second, metabolic complementation also refers to more complex scenarios where pathways can be fragmented and distributed between the members of the association (Price and Wilson, 2014; Van Leuven et al., 2014). Various examples of metabolic complementation in insect endosymbionts have been described in the past (Baumann et al., 2006; McCutcheon and Moran, 2011; Manzano-Marín and Latorre, 2016). As an example, some endosymbionts hosted by phloem-feeding insects (e.g., Buchnera aphidicola, Candidatus Tremblaya princeps, Candidatus Portiera aleyrodidarum) upgrade the host diet by supplying essential amino acids and vitamins absent in the diet (Zientz et al., 2004; Baumann et al., 2006; McCutcheon and von Dohlen, 2011). In other cases, as the cockroach endosymbiont Blattabacterium cuenoti, the assembly of metabolic reactions from the host and the symbiont allows the mobilization of the host nitrogen reservoirs (Patiño-Navarrete et al., 2014).

A remarkable case of endosymbiont consortia has been described in the cedar aphid Cinara cedri (Pérez-Brocal et al., 2006; Lamelas et al., 2011a). In this system, two species of endosymbiotic bacteria coexist. As is the case in most aphid species, the primary (obligate) endosymbiont is B. aphidicola $\mathrm{BCc}$, albeit in this insect species there is always a second (co-primary) endosymbiont, Candidatus Serratia symbiotica SCc (hereafter referred to as S. symbiotica SCc, GomezValero et al., 2004). The genomic analysis of this consortium has shown that many biosynthetic pathways are coded only in one of the two endosymbiont genomes, thus leading to obligate cross-feeding. Nonetheless, it is remarkable that the tryptophan biosynthetic pathway is split in two: Buchnera is able to synthesize up to anthranilate, whereas Serratia uses this anthranilate to synthesize tryptophan, which is required by all the members of the consortium (Gosalbes et al., 2008). The existence of metabolic complementations between endosymbionts and their host and, particularly, between the members of the bacterial consortium (as it is the case with the biosynthesis of tryptophan), poses several evolutionary questions: does complementation generate an adaptive advantage for the system as a whole? If this is the case, what is the nature of such advantage? Some theoretical studies have tried to illuminate whether the organisms exhibiting some degree of cooperation, as it is the case of cross-feeding, show any increase in their growth rate, compared to non-cooperative strains (Germerodt et al., 2016; Großkopf and Soyer, 2016). Furthermore, the metabolic pathway sharing between two endosymbionts has been suggested as a strategy to increase the efficiency of the biosynthesis of compounds when feedback inhibition is present in the pathway (Mori et al., 2016). Nevertheless, the emergence of such patterns of complementation poses diverse biophysical problems. For instance, the exchange of intermediates between endosymbionts implies a transport of solutes. It is well known that endosymbionts harbor a limited repertoire of transporters (Charles et al., 2011) and, on the other hand, the existence of membrane transporters specific for metabolic intermediates is very unusual. As a result, the exchanges should occur by simple diffusion and this situation imposes restrictions since metabolic intermediates usually show very low diffusion rates (Mori et al., 2016).

Another important matter in the evolution of endosymbiotic bacteria is the interplay between chance and necessity during the genome reduction process (Sabater-Muñoz et al., 2017). Although it is reasonable to accept the force of purifying selection, it is not clear if the patterns of complementation exhibited in these systems are the outcome of a random process, or if the observed patterns reflect an advantage over alternative evolutionary trajectories. In other words, to what extent are the evolutionary histories of these systems predictable? Insilico evolutionary experiments using genome-scale metabolic models (GEM) of two different endosymbionts (B. aphidicola and Wigglesworthia glossinidia) showed that the present gene content of these symbionts can be predicted with over $80 \%$ accuracy, from distant ancestors of the organisms and considering their current lifestyle (Pál et al., 2006). Similar studies have analyzed the fragility of the reduced metabolism to conclude that, in general, these networks cannot be further reduced (Pál et al., 2006; Thomas et al., 2009; Belda et al., 2012; González-Domenech et al., 2012; Ponce-de-Leon et al., 2013; Calle-Espinosa et al., 2016). A recent application of metabolic flux analysis to an endosymbiotic consortium has revealed distinct benefits and costs of the symbionts to their host (Ankrah et al., 2017), highlighting how the analysis of GEMs can be successfully applied to study consortia of uncultivable endosymbiotic bacteria.

Herein, we are interested in studying the interplay of chance and necessity in the evolution and emergence of metabolic complementation in endosymbiotic consortia. For this purpose, we have chosen the well-documented case of the consortium formed by the primary and co-primary endosymbionts of the cedar aphid, B. aphidicola BCc and S. symbiotica SCc. Individual GEMs have been reconstructed, manually curated and analyzed for each individual bacterium, based on their corresponding genome annotations. After extensive manual curation, these two models were used to create a compartmentalized consortium model named $i$ BSCc, which also include a set of key enzymatic 
activities performed by the host. We assessed the metabolic connections between the two endosymbionts and with the host to predict patterns of metabolic complementations. We observed that the combination of these two extremely reduced metabolic networks results in an integrated yet fragile system. Finally, we performed in-silico evolutionary experiments to study the paths leading to the emergence of metabolic complementation.

\section{MATERIALS AND METHODS}

\section{Annotated Genomes}

In order to reconstruct the genome-scale metabolic models (GEM) of S. symbiotica SCc and B. aphidicola BCc, we retrieved the corresponding genomes and the semi-automatically reconstructed Pathway-Genome Databases (PGDB) available in the BioCyc collection in Caspi et al. (2014). The used PGDBs' versions were SSYM568817 19.0 for S. symbiotica SCc, and BAPH37261 19.0 for B. aphidicola BCc (both available in Tier 3 at BioCyc 19.0) (see Supplementary Text S2). The public version of BAPH372461 does not include the information encoded in the pTpr-BCc plasmid (accession number EU660486.1, Gosalbes et al., 2008). This plasmid contains two genes $(\operatorname{trp} E$ and $\operatorname{trp} G)$ (Table 1), and was manually added to a local version of the $B$. aphidicola BCc PGDB, created through Pathway Tools v. 19.0 (Karp et al., 2015). Once added, the PathoLogic algorithm was run again in order to update the pathway prediction.

TABLE 1 | Enzymatic activities and genes involved in the biosynthetic pathways of tryptophan, THF and biotin as grouped in 7 enzyme subsets (ES).

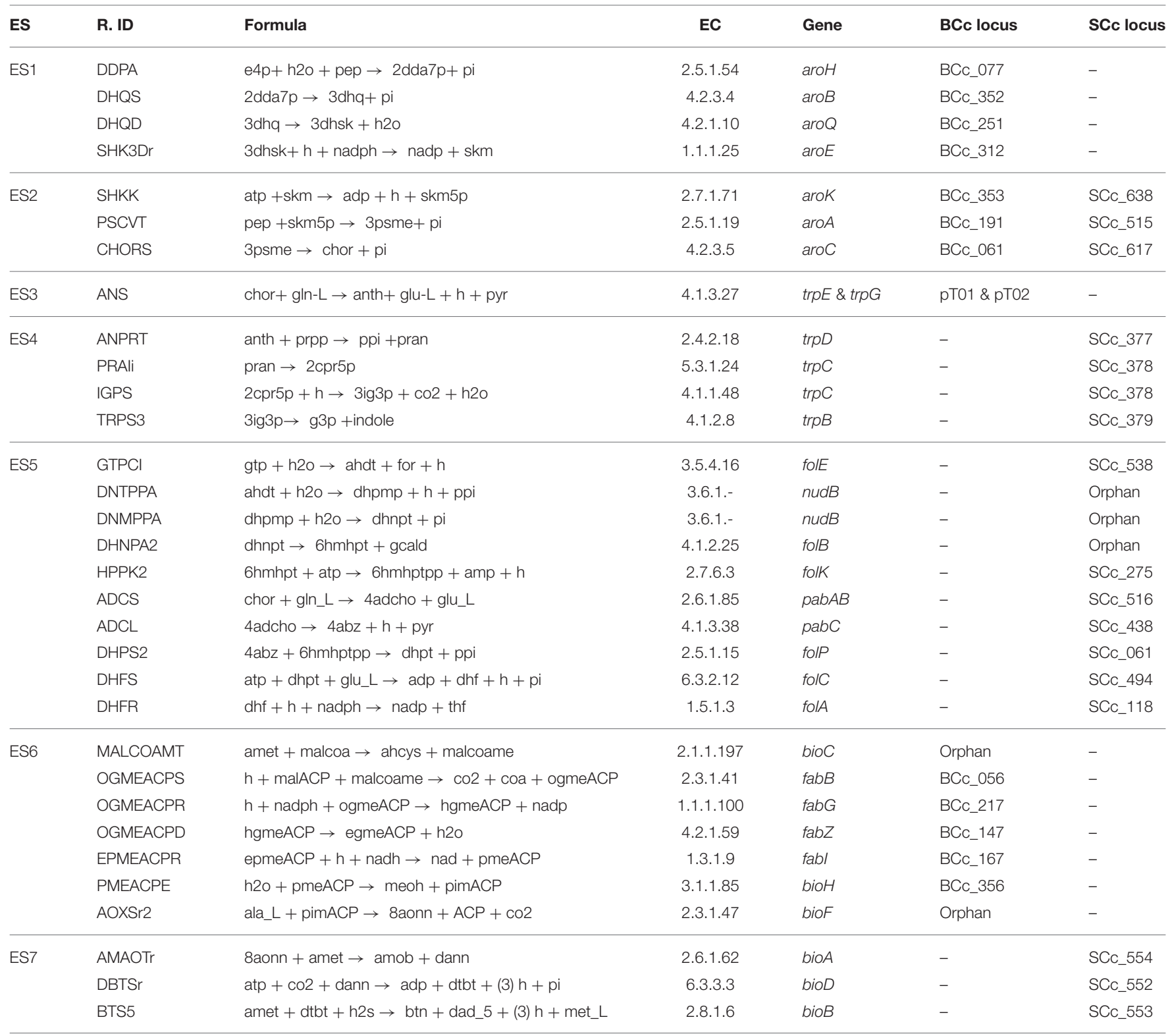

IDs for reactions and metabolites are as obtained from the BiGG database. 


\section{Reconstruction and Refinement of the Metabolic Models}

The reconstruction and refinement of the metabolic models was performed following the protocol described by Thiele and Palsson (2010). In order to reconstruct the metabolism of S. symbiotica SCc, the GEM of E. coli K12 MG1655 iJO1366 (Orth et al., 2011) was used as a reference, since this is the phylogenetically closest free-living organism for which a highly refined and validated model exists. First, orthologous genes were identified between the E. coli K12 MG1655 genome (available in EcoCyc 19.0) and the S. symbiotica SCc genome included in the PGDB SSYM568817 19.0. After the gene sequences were extracted and translated, they were compared using Blastp (Altschul et al., 1990) with an e-value maximum of $10^{-10}$ and an identity minimum of $75 \%$. Subsequently, this proteome-level comparison was used to infer orthology using OrthoMCL (Li et al., 2003). Combining these results with the set of reactions and pathways present in the SSYM568817 PGDB, a gene-protein-reaction (GPR) table was constructed. Using this GPR table, together with the iJO1366 model and the BiGG database (Schellenberger et al., 2010) a first version of the S. symbiotica SCc model was reconstructed. Moreover, the biomass equation introduced in the model is a modified version of the one present in iJO1366, from which we removed membrane components and cofactors absent in the S. symbiotica SCc network. The coefficients were corrected using the methodology suggested by Henry et al. (2010). The first draft obtained was revised combining the unconnected module (UM) approach (Ponce-deLeon et al., 2013) together with the previously published genome analyses (Lamelas et al., 2011a). Regarding the metabolism of $B$. aphidicola BCc, a previously published GEM, named BCc (Belda et al., 2012), was used as a reference. However, this GEM contained several blocked reactions and dead-end metabolites. The resolution of the different gaps was performed by solving the set of UMs combining the pathway inferences present in the BAPH372461 PGDB and the GEM of $B$. aphidicola Bap (MacDonald et al., 2011), available in BioModels (MODEL1012300000).

\section{Construction of a Biomass Equation for the Aphid C. cedri}

The aphid biomass equation was defined by including the various biomass components in corresponding stoichiometric proportions. Since the consortium model does not include the metabolism of the host, the aphid biomass equation only includes the compounds that are provided by the endosymbiotic consortium (i.e., the essential amino acids, vitamins, and cofactors) as previously done (Calle-Espinosa et al., 2016). The stoichiometric coefficients for the set of amino acids provided by the endosymbionts were estimated from the analysis of composition of the Aphis fabae and Acyrtosiphon pisum proteomes (Douglas et al., 2001; Russell et al., 2014). The compositions measured in two different aphid species showed good agreement, a fact that suggests that the values can be extrapolated to C. cedri (see Supplementary Text S1).
We used the values from the experimental measurements of the A. pisum amino acid composition obtained by Russell et al. (2014) because this dataset includes the measurements of certain amino acids not included in Douglas et al. (2001). The values were normalized to represent composition in $1 \mathrm{~g}$ of dry weight (DW) in the aphid biomass. Additionally, the cofactors provided by the endosymbiotic consortium to the host were added. Since there is no estimation of their proportion in the aphid biomass, their values were set to be several orders of magnitude below the amino acids demand, but reflecting their essentiality to the host (Supplementary Table S5).

\section{Constraint-Based Modeling Methods}

The different constraint-based methods used in the present work correspond to the current implementation found in COBRApy toolbox 0.6 (Ebrahim et al., 2013). The boundary conditions used in the individual analyses of the endosymbionts models, iSCc226 and $i$ BCc98, can be found in Supplementary Tables S1, S2, respectively. However, in the case of the compartmentalized model of the consortium, a restriction that limits the total number of carbon atoms diffusing through the membrane of each endosymbiont was introduced (Burgard et al., 2001). By doing so, we are able to separately constrain the exchange of metabolites of each endosymbiont with its surrounding environment without the need of establishing arbitrary boundary for each exchange flux. Thus, instead of having a large set of parameters, one for each exchange flux, we only used two parameters, one for each endosymbiont. The restriction can be expressed as follows:

$$
\sum_{i \in T_{k}} v_{i} . n c_{i} \leq U B \forall k \in\{B C c, S C c\}
$$

Where $T_{k} \subset J$ is the set of indexes of the transporters present in the compartment $\mathrm{k}$ (endosymbiont) and $J$ the set of all fluxes; $v_{i}$ is the flux through the transporter $i ; n c_{i}$ is the number of carbon atoms in the transported molecule; and UB is the upper bound, i.e., the parameter establishing the maximum number of carbon atoms that can be exchanged by the $\alpha$ compartment (one of the endosymbionts) and the extracellular compartment (the host cell). This parameter limits the amount of matter flowing through the membrane in terms of the total carbon atoms and was fixed to 100 carbon atoms. In order to guarantee that the transport fluxes are non-negative, the reversible transporters were split into two irreversible transport reactions with opposite direction (Burgard et al., 2001). The biosynthetic capabilities of the endosymbionts for each biomass component, as well as for energy production, were assessed using Flux Balance Analysis (FBA) (Orth et al., 2010). Furthermore, network fragility was predicted through in-silico knockout experiments conducted using FBA as well as the Minimization of the Metabolic Adjustment (MOMA) (Segrè et al., 2002). Details on each method can be found in the extended materials and methods (see Supplementary Text S2). 


\section{RESULTS}

\section{Metabolic Reconstruction of the Cedar Aphid Primary and Co-primary Endosymbionts}

The metabolic models of S. symbiotica SCc and B. aphidicola $\mathrm{BCc}$ were reconstructed individually, and were named iSCc236 and $i \mathrm{BC} 988$, respectively (see Supplementary Text $\mathbf{S 1}$ for further details on the reconstruction, and Supplementary Tables S1, S2 and Supplementary File $\mathbf{S 1}$ for the complete models). The former consisted of 267 intracellular metabolites and 209 reactions catalyzed by the products of 236 genes, plus 11 orphan reactions (i.e., reactions with unknown coding genes). It also includes 30 transporters associated with a gene, and 49 orphan transport reactions. On the other hand, $i \mathrm{BC} 98$ yielded a smaller network, containing 155 intracellular metabolites and 95 reactions catalyzed by the products of 98 genes, and 8 additional orphan reactions. Additionally, it includes only 5 transporters associated with a gene, and 58 orphan transporters. In both cases, all orphan reactions are required by the model in order to predict biomass formation. The genomes of these endosymbionts contain only a small set of genes coding for substrate-specific transport systems (Wernegreen, 2002; Charles et al., 2011). However, the corresponding metabolic models predict the necessity of metabolite transit through the endosymbiont membrane, albeit the transport mechanisms have not been elucidated in many cases, in which simple diffusion has been proposed as a plausible mechanism (Mori et al., 2016). The metabolic requirements and the biosynthetic capabilities for each of these two models were congruent with those inferred from genomic analyses (Lamelas et al., 2011a), with the exception of the biosynthesis of asparagine, which is predicted by the iSCc236 model to be required as nutritional input provided by the host instead of being synthesized by S. symbiotica SCc (Supplementary Figure S1). Finally, when the energetic capabilities, i.e., the synthesis of ATP, were analyzed for both symbionts, we found that $i \mathrm{BCc} 98$ predicts a very low yield of ATP, a limitation that is a direct consequence of the absence of ATP synthase. In turn, this lack of a proton pump mechanism poses a constraint on the regeneration of NADH, through the $\mathrm{NADH}$ dehydrogenase complex. The model suggests that part of the NADH may be driven through the conversion of the pair homocysteine and serine, into glycine and methionine (for further details, see Supplementary Text S1).

Since we were interested in modeling the whole consortium, the two metabolic models previously introduced ( $i \mathrm{BC} c 98$ and iSCc236), were combined to create a single model named $i \mathrm{BSCc}$. Although it is known that B. aphidicola BCc population and S. symbiotica SCc are hosted in different bacteriocytes, a more simplistic representation was chosen where both endosymbiont models are embedded in a single compartment, in a similar way as it has been previously done (Ankrah et al., 2017). Therefore, our model included: (i) a compartment representing the $B$. aphidicola $\mathrm{BCc}$ population; (ii) a compartment representing the S. symbiotica SCc population; and (iii) a single extracellular compartment representing the host cells, where both symbionts are embedded (Figure 1). Consequently, the boundary of the system was defined by the compartment representing the host, and exchange fluxes across the boundary represented the metabolites supplied and consumed by the aphid, as well as the excretion products. The 52 metabolites found in the extracellular compartment include those obtained from the host, common excretion products and metabolites exchanged by the two symbionts. Additionally, five reactions were added to the extracellular compartment, as they have been suggested to be performed by the host and to play a relevant role in the metabolic complementation between host and symbionts (Hansen and Moran, 2011; Poliakov et al., 2011). These reactions include the conversion of phenylalanine into tyrosine (1 reaction), the production of homocysteine and adenosyl-methionine from cysteine (3 reactions), and the assimilation of hydrogen sulfide produced by S. symbiotica SCc, for the production of lipoate (1 reaction) (see Supplementary Table S3).

In order to simulate the growth of the system as a whole, we introduced a combined meta-biomass equation, where each member contributes to the growth of the system with a fixed stoichiometry (Figure 1A). Our model assumes the coupling between the growths of all the members of the system, which would especially apply during the development of the host (Figure 1B). This assumption is justified by different sources of experimental data as well certain theoretical results (see Supplementary Text $\mathbf{1}$ for further details). Since the exact contribution of the symbionts to the cedar aphid biomass is unknown, we used data obtained from Schizaphis graminum and A. pisum indicating that their symbionts represent 5$15 \%$ of the system's biomass (Whitehead and Douglas, 1993; Baumann et al., 2006). Thus, we modeled the proportion between biomass of the symbionts and the host to be 1:9. Finally, since imaging data from the cedar aphid bacteriome indicates similar proportions between the two symbiont species (Gomez-Valero et al., 2004; Pérez-Brocal et al., 2006), we modeled that each bacterial member represents $5 \%$ of the system's biomass at every time. It is important to emphasize that this assumption is key for the direct utilization of FBA and other related techniques in the study of the $i \mathrm{BSCc}$ model. The reason is that, if both symbionts are in proportion 1:1, the fluxes are normalized by the same quantity (e.g., dry weight of bacteria) and they grow necessarily at the same growth rate. This means that they behave essentially as one "big" bacteria. However, if this assumption does not hold, and the growth rate of the two bacteria are the same (i.e., they are coupled), new (probably non-linear) constraints should be incorporated to the model (see Kerner et al., 2012 as an example). Once the metabiomass equation was introduced, we use FBA to verify that the $i \mathrm{BSCc}$ model is consistent, allowing for a positive metabiomass flux and the growth of each member of the consortium (see Supplementary Table S3). This optimal flux distribution predicted by FBA (maximization of the meta-biomass, i.e., the growth rate of the $i \mathrm{BSCc}$ consortium) is analyzed in the next section.

\section{Metabolic Analysis of the Cedar Aphid Consortium}

The overall structure of the models corroborates that the metabolic network of $B$. aphidicola $\mathrm{BCc}$ is specialized in the production of amino acids, while S. symbiotica SCc 


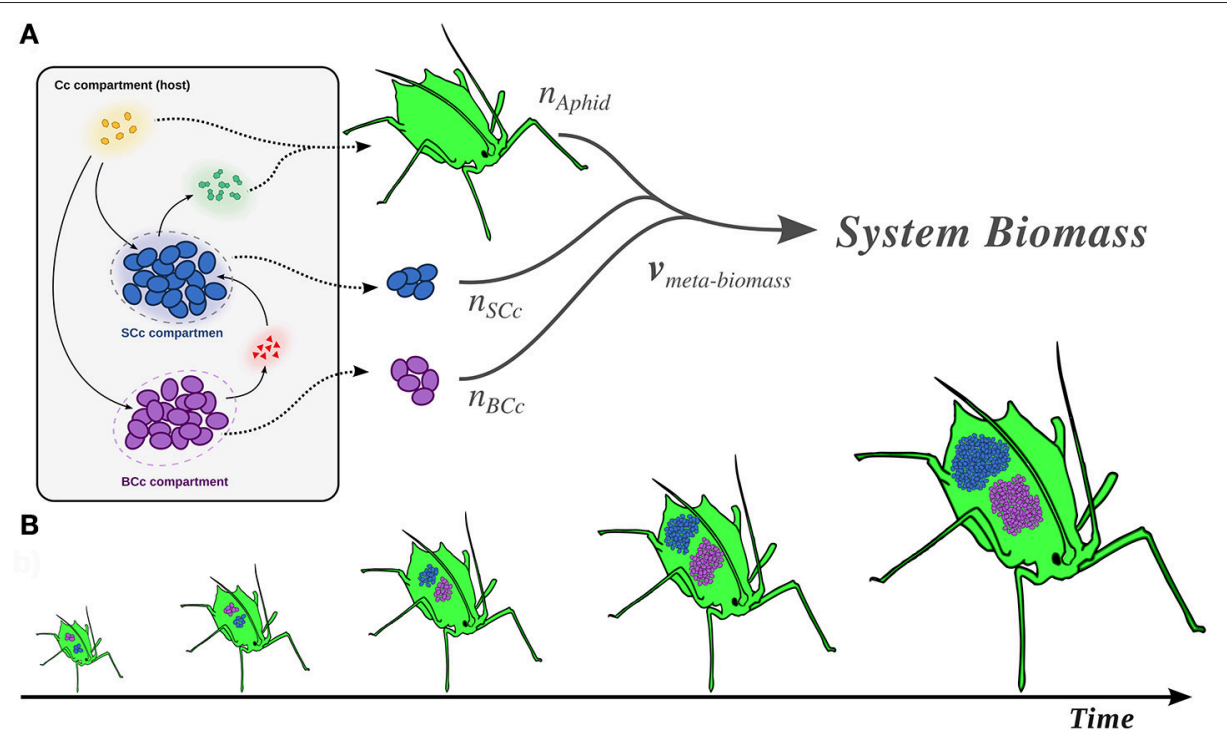

FIGURE 1 | Compartment modeling of the cedar aphid endosymbiotic consortium and meta-biomass equation modeling the growth of the endosymbiotic consortium and the host. (A) Representation of the three compartments model of the endosymbiotic consortium. Dotted arrows represent the biomass production of each member. The thick tripartite arrow represents the system biomass, i.e., the meta-biomass (see main text). The annotations $n_{\alpha}$ with $\alpha \epsilon\{B C c$, SCc, Aphid\} correspond to the stoichiometric contribution of each member to the total system biomass equation. Solid arrows: transport processes within the system (e.g., metabolic complementation). (B) Coupling between the growth of the members in the system, representing stability in the ratio of their biomass.

produces nucleotides and a large number of cofactors (Supplementary Figure S1). Moreover, the model predicts that S. symbiotica SCc can synthesize tryptophan from anthranilate, which has been shown to be provided by $B$. aphidicola $\mathrm{BCc}$ in a paradigmatic case of metabolic complementation (Gosalbes et al., 2008; Lamelas et al., 2011a; Martínez-Cano et al., 2015; Manzano-Marín et al., 2016). Another case of metabolic complementation between the two symbionts predicted by the model occurs in the biotin synthesis pathway, which takes place via the import of the precursor 8-amino7-oxononanoate (8AONN), produced by B. aphidicola $\mathrm{BCc}$, as recently suggested from genomic data (Manzano-Marín et al., 2016). Lysine biosynthesis also represents a case of complementation, based on the fact that the genome of $S$. symbiotica SCc encodes all activities of the pathway except the last one, which converts meso-diaminopimelate into lysine (Lamelas et al., 2011a). However, our model indicates that meso-diaminopimelate is used for the synthesis of peptidoglycan, while the complete lysine synthesis pathway is conserved in $B$. aphidicola $\mathrm{BCc}$, suggesting that this complementation does not occur.

The FBA predictions showed that B. aphidicola BCc synthesizes and provides the host and S. symbiotica SCc with ten amino acids. B. aphidicola BCc also synthesizes anthranilate, $8 \mathrm{AONN}$ and shikimate and releases them to the host compartment. In turn, S. symbiotica SCc imports anthranilate, $8 \mathrm{AONN}$ and shikimate, and use them as precursors for the biosynthesis of tryptophan, biotin and tetrahydrofolate (THF), respectively (see Figure 2 and Supplementary Table S3). Therefore, three events of metabolic complementation between the two symbionts are predicted, including the biosynthesis of tryptophan, biotin and THF; whereas the former two have been inferred previously from genomic analyses (Gosalbes et al., 2008; Manzano-Marín et al., 2016), the latter is a redefined complementation case (Lamelas et al., 2011a, see Figure 2). Folate cross-feeding has been described between Serratia grimesii and Treponema primitia, both members of the termite Zootermopsis angusticollis gut microbiome (Graber and Breznak, 2005). Since C. cedri, as animals in general, is not able to synthesize folate, and this is not included in the aphid's diet, Serratia probably provides this essential cofactor to both the host and Buchnera.

Regarding S. symbiotica SCc, the $i$ BSCc model predicts that it can synthesize, without need for complementation, cysteine, the four deoxynucleotides, the four triphosphate nucleosides, and thirteen cofactors and coenzymes. Several of these compounds may also be synthesized by the host (e.g., nucleotides, deoxynucleotides and $\mathrm{NAD}+$ ), who might provide them to B. aphidicola BCc. Indeed, the aphid A. pisum, as most eukaryotes, is able to synthesize nitrogenous bases (Richards et al., 2010; Vellozo et al., 2011), which indicates that C. cedri also should. In this study, however, we will assume that it is S. symbiotica SCc who provides Buchnera with nucleotides and cofactors, and the host with cofactors such as biotin, riboflavin and THF.

\section{Fragility Analysis: Can the Networks Be Further Reduced?}

The construction of metabolic models of two different endosymbionts with a notable difference in size allowed us to study whether the reductive evolutionary trends indeed generate smaller, more fragile networks. The robustness of the 

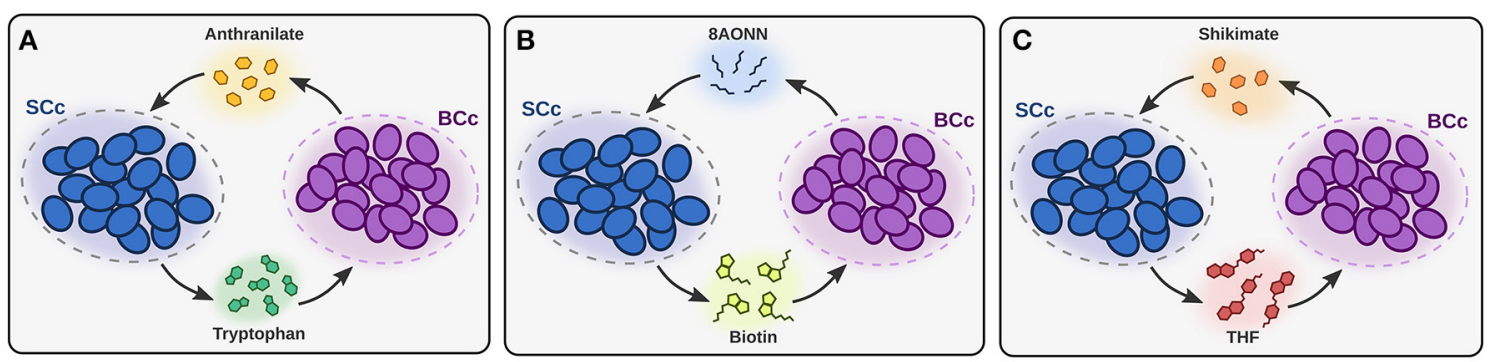

FIGURE 2 | Patterns of metabolic complementation predicted by iBSCc. In (A) the complementation for tryptophan biosynthesis, where BCc produces anthranilate and SCc uses it to produce tryptophan, is shown. In $\mathbf{B}, \mathbf{C})$ the complementation for the biosynthesis of biotin and THF, respectively, are shown.

metabolic networks of the cedar aphid endosymbionts was assessed through in-silico knockout analyses conducted with two alternative approaches: FBA and MOMA. In the first place, the fragility of the whole consortium was considered by using the meta-biomass flux as an indicator of the viability. The results show that around $85 \%(\sim 88 \%$ using MOMA) of the genes coded by the endosymbionts are essential in order to sustain the growth of the whole system (see Supplementary Table S4). Then we focussed on the fragility of the individual endosymbionts networks. For the case of $i \mathrm{BCC} 98 \mathrm{FBA}$ predicted that 72 out of the 98 metabolic genes $(\sim 74 \%)$ are essential, while the MOMA analyses identify $76(\sim 78 \%)$ as essential. The dispensable genes are mostly involved in catabolism, affecting the phosphate pentose pathway, glycolysis, respiratory chain and pyruvate fermentation (see Supplementary Table S4). When performing FBA robustness analyses on iSCc236, 209 genes $(\sim 88 \%)$ are predicted to be essential, whereas MOMA predicted 5 additional genes as essentials. The 28 dispensable genes predicted by both methods code for 36 enzymatic reactions involved mostly in biosynthetic pathways (e.g., nucleotides and cofactors), but also in the central carbon metabolism (e.g., the pentose phosphate pathway and glycolysis). If we consider the cell wall genes to be dispensable (since they have been repeatedly lost in endosymbiotic bacteria), the number of essential genes drops to $189(\sim 80 \%)$. Additionally, if it is also assumed that the host is who provides the nucleotides and deoxynucleotides, the percentage of essential genes drops to $\sim 70 \%$ (data not shown). Although it might seem surprising that these estimates are higher or comparable to those obtained by using the smaller $i \mathrm{BCc} 98$ network, the iSCc236 model requires 22 organic compounds to be imported, while $i \mathrm{BCc} 98$ requires 29 , among which there are nucleotides and cofactors such as $\mathrm{NAD}^{+}$and coenzyme A. Finally, a study of distributed robustness was performed through the analysis of the synthetic lethal (or double lethal) genes, i.e., pairs of non-essential genes whose simultaneous inactivation yields lethality (Wagner, 2005). In the case of $i \mathrm{BCc} 98$, ca. $15 \%$ of the pairwise combinations between the 26 non-essential genes predicted with FBA are predicted as synthetic lethal. These combinations include 20 of the non-essential genes, indicating that most of the dispensable genes have only a shallow degree of redundancy. On the other hand, iSCc236 predicts only ca. $5 \%$ of the possible combinations between the 34 non-essential genes to be lethal. Moreover, by disregarding the cell wall biosynthesis genes as explained above, this number drops to ca. $2 \%$.

\section{In-Silico Reduction Experiments: Evaluating Alternative Evolutionary Scenarios}

Although it is generally accepted that these patterns of complementation are the outcome of the process of genome reduction, whether such organization of the metabolic networks confer a selective advantage to the whole system or not, remains an open question. Previous work has focused on the kinetic aspect of the problem, in particular the role of product inhibition as a plausible condition that may drive the emergence of metabolic complementation (Mori et al., 2016). Herein, we try to approach this problem from a structural point of view, by comparing the metabolic capabilities in alternative scenarios of gene losses and retentions within the three-shared pathways. For this purpose, $i \mathrm{BSCc}$ was extended to represent a putative ancestral-state model of the consortium, named $i \mathrm{BSCc}_{\text {Ancest }}$, where the three-shared pathways are still complete in both endosymbionts (see Supplementary Text S2). In this way, it is possible to compare how the different scenarios of gene loss and retention perform with respect to the putative ancestor as well as to the pattern of complementation exhibited by the cedar aphid consortium.

Using $i \mathrm{BSCc}_{\mathrm{Ancest}}$, the space of all viable and scenarios of gene loss and retention patterns (GLRPs) were generated by removing from this model every possible combination of genes, from single genes to the most reduced cases where only one copy of each gene remains present (at least one of the two copies for each gene needs to be functional). A GLRP is considered viable if, after removing the corresponding reactions, FBA predicts a meta-biomass flux greater than zero. Furthermore, in order to reduce the number of combinations of possible GLRPs, the enzyme subsets (ES), i.e., groups of enzymes that always work together under steady state, were first computed for $i \mathrm{BSCc}_{\mathrm{Ancest}}$ (see the extended Materials and Methods section in the Supplementary Text S2). Since removing an enzyme from an ES is equivalent to removing all the enzymes in the ES, each ES can be treated as a functional unit. Then, those genes coding for enzymes in the same ES 
were grouped together. Table 1 shows the structure of the ES for the three pathways considered in this study. Notably, the 32 enzyme activities are grouped in 7 ESs. Given that in $i \mathrm{BSCc}_{\text {Ancest }}$ each endosymbiont includes the $7 \mathrm{ESs}$, the enumeration process yielded a total of 2188 viable GLRPs, which represent alternative consortium models. From this set, 128 are minimal GLRPs, i.e., only one copy of each gene remains active. It is worth noting that the pattern exhibited by the C. cedri consortium is not minimal since the genes coding for the three activities which allow the conversion of shikimate into chorismate are present (see Table 1). In order to simplify the notation each GLRP was coded by a sequence of symbols $\Delta_{n}^{m}$, where the subscript $n$ (from 1 to 7 ) denotes the loss of the enzyme subset by one of the endosymbionts, indicated by the superscript $m$, which can be either $B$ or S (B. aphidicola or S. symbiotica). Accordingly, $\Delta_{1}^{B}$ represents a simple GLRP where $B$. aphidicola has lost the ES1. A more complex example would be the case of $i$ BSCc (which is the actual pattern exhibited by B. aphidicola and $S$. symbiotica in the cedar aphid). In this case, the GLRP is coded as follows: $\Delta_{1}^{S} \Delta_{3}^{S} \Delta_{4}^{B} \Delta_{5}^{B} \Delta_{6}^{S} \Delta_{7}^{B}$. Note that in this case the ES2 is omitted because the genes involved in this ES are present in both endosymbionts, as mentioned above (see Table $\mathbf{1}$ ).

The 2188 consortium models, which represent alternative evolutionary scenarios, were evaluated using FBA in two ways by considering: (i) the individual maximal production rate of tryptophan $\left(v_{T r p}^{*}\right)$, THF $\left(v_{T H F}^{*}\right)$ and biotin $\left(v_{B t n}^{*}\right)$; and (ii) the whole system performance, calculated by optimizing the metabiomass production rate. In all the cases, the optimal values are normalized with respect to the optimal value showed by $i \mathrm{BSCC}_{\mathrm{Anc}}$. Figure 3 summarizes the results of the reduction experiment in terms of the production capabilities of tryptophan, THF and biotin for each GLRP. Firstly, the results show that for any of the three objectives, the production rates of the different GLRPs exhibit great variability (for more details see Supplementary Table S5). This clearly shows that the way in which a pathway is distributed in a complementation event has a profound impact in the pathway capabilities. In the case of the tryptophan production, the different GLRPs can be divided into two main groups: (i) a group of GLRPs (which includes $i \mathrm{BSC}$ ) with a tryptophan production rate almost equal to the one exhibited by the putative ancestor (i.e., $v_{\operatorname{Trp}}^{*} \sim 100 \%$ ); and ii) a larger group of GLRPs with production rate $v_{T r p}^{*}<90 \%$ (Figure 3a). Furthermore, when considering only the minimal GLRPs (i.e., cases with $50 \%$ of genes lost) the gap is even larger, and there is only one GLRP with $v_{\operatorname{Trp}}^{*} \sim 100 \%$ whereas the other patterns exhibit a $v_{\operatorname{Trp}}^{*}<76 \%$. This minimal GLRP with $v_{\operatorname{Trp}}^{*} \sim 100 \%$ corresponds to the pattern exhibited by $i$ BSCc with the additional loss of the only set of redundant genes that remain present, which form the ES2 (see Table 1).

On the other hand, when considering the biosynthesis of THF and biotin, the results also show a wide dispersion for the normalized production rate values exhibited by the different GLRPs. However, unlike the case of the tryptophan, in these two cases the optimal production value exhibited by $i \mathrm{BSC}$ decreases considerably with respect to the value exhibited by the putative ancestor (Figures $\mathbf{3 b}, \mathbf{c}$ ). For the case of the production of THF, all the GLRPs with the highest normalized production rate $\left(v_{T H F}^{*} \sim 93 \%\right)$ and with more than $40 \%$ of the genes lost, shared the following two losses: $\Delta_{1}^{S}$ and $\Delta_{5}^{S}$, which correspond to the scenario in where $S$. symbiotica losses the biosynthetic pathways of shikimate and THF. Whereas $\Delta_{1}^{S}$ is consistent with the GLRP shown by $i$ BSCc, $\Delta_{5}^{S}$ is the opposite, i.e., in the cedar aphid consortium B. aphidicola has lost the THF biosynthetic pathway. Furthermore, all those GLRPs that involve $\Delta_{5}^{B}$ imply an important drop in the normalized production rate $v_{T H F}^{*}<$ $72 \%$ (Figure $3 \mathbf{b}$ ). In the case of the biotin biosynthesis where the GLRP of $i$ BSCc implies $v_{B t n}^{*} \sim 64 \%$ something similar was found (Figure 3c). According to these results, when the system has lost more than $40 \%$ of the genes involved in the analyzed pathways, those GLRPs with a biotin production rate closest to the one exhibited by the putative ancestor, share the same pattern $\left(\Delta_{6}^{B} \Delta_{7}^{S}\right)$. However, this pattern is opposite to the one exhibited by $i \mathrm{BSCc}$, i.e., $B$. aphidicola losses the capability to synthesize $8 \mathrm{AONN}$ and retains the capability to produce biotin from this precursor, whereas S. symbiotica
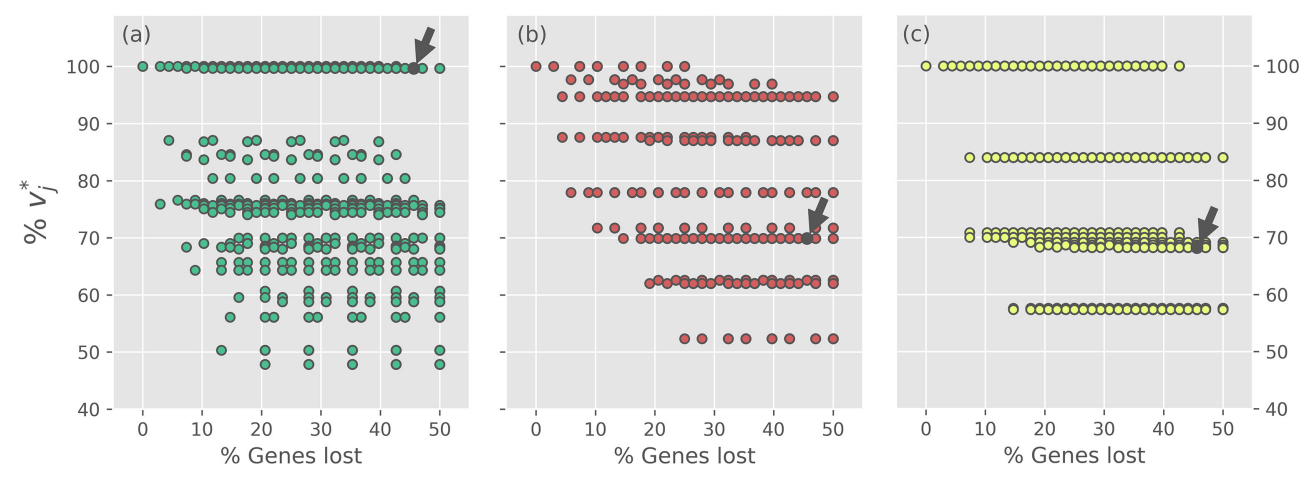

FIGURE 3 | Optimal production rates of tryptophan, THF and biotin for the different gene loss and retention scenarios. In each panel, the normalized optimal production rate $v_{j}^{*}$ is plotted against the percentage of gene losses, for every gene loss scenario. From (a-c) the panels correspond to the optimization of the rate $v_{j}^{*}$ of production of tryptophan, THF and biotin, respectively (i.e., $v_{T r p}^{\star}, v_{T H F}^{\star}, v_{B t n}^{\star}$ ). For each target, optimal production rates are normalized with respect to the optimal

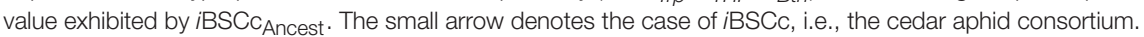


exhibits the complementary pattern. Similar results were found when considering the reduction of each pathway individually, i.e., when only a single pathway is considered redundant, the GLRPs include only the genes involved in the pathway (see Supplementary Figures S2-S4 and Supplementary Table S6).

After the analysis of how the different GLRPs perform over the production rates of individual biomass components, the same study was conducted but using the meta-biomass production rate as a proxy to study the fitness of the alternative evolutionary scenarios. The simulation results, summarized in Figure 4, show that the value of the normalized meta-biomass production $\left(v_{\text {meta-biomass }}^{*}\right)$ varies between 95 and $100 \%$, compared to the putative ancestor (Figure 4a). Although this range is quite narrow (Figure $4 b$ ), it may still play a role in a selective process, since herein only the stoichiometric rate is considered, and no other factor, such as the cost of protein synthesis, is considered. Moreover, in a first look the results indicate that the iBSCc GLRP $v_{\text {meta-biomass }}^{*}$ is quite close to the one of the putative ancestor.

A deeper insight into the results shows that among the minimal GLRPs, 48 out of the total $128(\sim 37 \%)$ also exhibit a $v_{\text {meta-biomass }}^{*}>99 \%$. Furthermore, within this set it is possible to find opposite patterns, for example cases such as $\Delta_{3}^{S} \Delta_{4}^{S}$ and $\Delta_{3}^{B} \Delta_{4}^{B}$ imply almost identical production rates. Nevertheless, there are some particular patterns that are consistent with the results obtained analyzing the individual pathways. For example, all the minimal GLRPs which include the loss $\Delta_{3}^{B} \Delta_{4}^{S}$ (i.e., the opposite pattern than the exhibited by $i \mathrm{BSCc}$ ) imply $v_{\text {meta-biomass }}^{*}<99 \%$. On the other hand, there are many GLRPs that could represent disadvantages in terms of the system's growth rate. Such would be outcompeted by other more efficient organization of the complementation. Thus, considering only the structure of the networks of the endosymbionts, the simulations indicate that although many GLRPs are possible, the pattern observed in the cedar aphid consortium has retained a large growth rate while undergoing a large degree of gene loss.

\section{DISCUSSION}

\section{Metabolic Consequences of Genome Reduction}

The reconstruction and metabolic analysis of $B$. aphidicola $\mathrm{BCc}$ and S. symbiotica SCc, co-primary endosymbionts of the cedar aphid C. cedri, has allowed, in first place, the revision of the annotation of these organisms' genomes. Indeed, through the manual curation of these networks using the UM approach, it was possible to correct annotation errors in different enzymatic activities, and to identify previously unannotated metabolic genes. Moreover, the simulations performed here allowed the refinement of growth conditions and metabolic capabilities of these endosymbionts as compared to previous inferences from genomic analyses. Our study also indicates that GEMs are a possible way to approach the otherwise inaccessible phenotypic features of uncultivable endosymbionts. On the other hand, the analysis of highly reduced metabolic networks, such as the case of $i \mathrm{BCc} 98$, bring into consideration methodological issues such as the case of the proton balance. Previously, a sensitivity analysis by Reed et al. (2003) in the genome-scale metabolic model of E. coli K12 iJR904 revealed that the net proton balance could be positive or negative depending on the carbon source used in the growth simulations, which would acidify or basify the environment, respectively. Alas, these predictions remain empirically untested.

The effect caused by proton balance in GEMs is generally low due to the size of these networks. However, in endosymbionts and other small networks, it may generate notorious consequences.

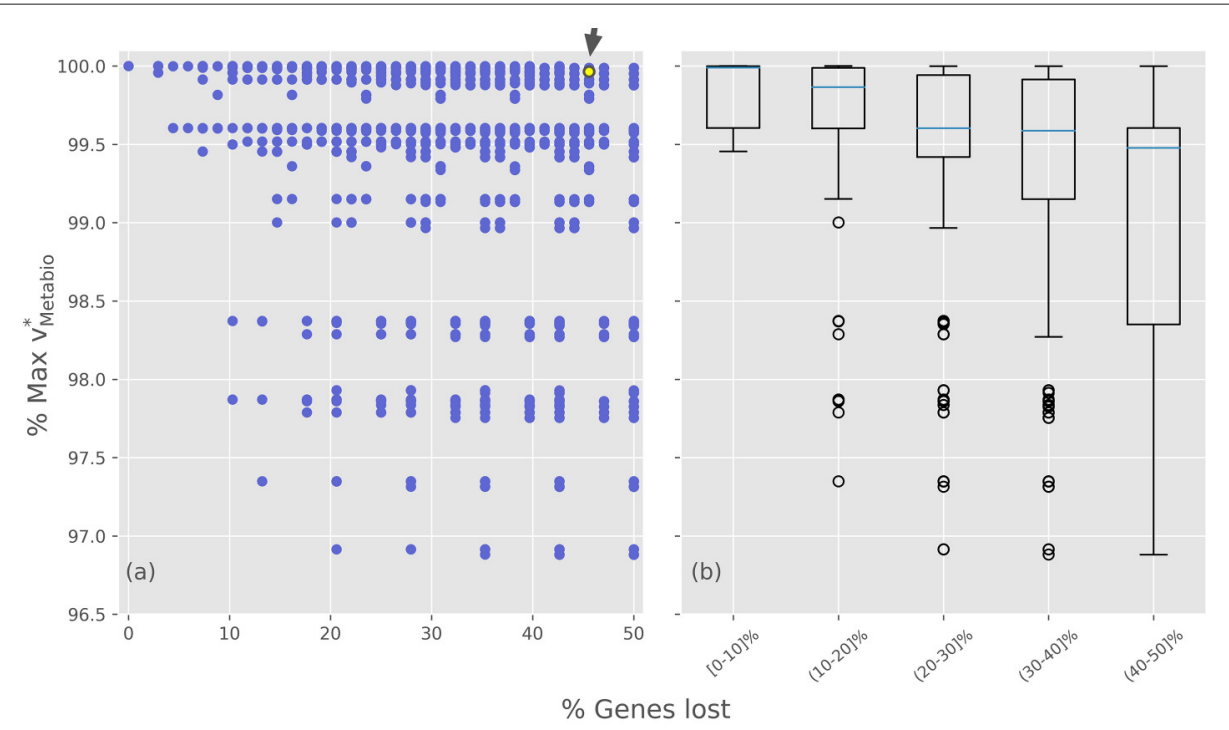

FIGURE 4 | Optimal meta-biomass production rates for the different gene losses and retention scenarios. (a) Normalized optimal meta-biomass production rate plotted against the percentage of gene loss for each GLRP. (b) Different GLRPs were grouped by intervals of gene losses. The small arrow denotes the case of iBSCc, i.e., the cedar aphid consortium. 
In $i \mathrm{BCc} 98$, for instance, it considerably reduces the versatility of the metabolism of $B$. aphidicola BCc by coupling presumably independent processes, such as ATP synthesis and the folate cycle, and over-producing amino acids. Although this metabolic organization would be clearly disadvantageous for a freeliving organism, for an endosymbiont member of a nutritional symbiosis it may be selected for at the level of the host. Indeed, a similar behavior has been described recently as applied to the whitefly endosymbiont $P$. aleyrodidarum (Calle-Espinosa et al., 2016), where the growth of the organism is coupled with the overproduction of amino acids and carotenoids as a consequence of its low energetic capabilities. This phenomenon might play a relevant role in the evolution of nutritional endosymbiosis but it may also represent a methodological artifact as the consequence of a lack of knowledge on how to formulate in such a model the transport of protons through the membrane. One possibility would be the use of protons for the transport of compounds against their gradients. Although the scarcity of annotated transporters in the B. aphidicola BCc genome (Charles et al., 2011) does not seem to support this scenario, this problem falls within a more general umbrella, whereby the nature of the cell envelope (including both the membrane composition and the transport systems) of endosymbionts is largely unknown and might rely on contributions from the host (McCutcheon, 2016).

On the other hand, simulations with iBCc98 and iSCc236 indicate resemblance with previous metabolic analyses from endosymbionts and other bacteria with reduced genomes. We found that these two networks contain very few dispensable genes, with essentiality estimates being around $88 \%$ of genes for iSCc236, and $73 \%$ for $i \mathrm{BCc} 98$ (91 and 78\%, respectively, according to MOMA). Although, these results may contradict the idea that the smaller the network, the higher the essentiality of its components, the percentage of essential genes predicted by iSCc236 drastically drops to $\sim 70 \%$, when the cell wall genes are considered dispensable, and that the nucleotides and deoxynucleotides are provided by the host. Moreover, a further 15 and $5 \%$ of the genes, respectively, are genes that become essential after the deletion of another non-essential gene, thus displaying only a shallow degree of redundancy. Altogether, the amount of non-essential genes seems to positively correlate with the size of the network (Gil and Peretó, 2015). Moreover, a previous metabolic analysis of $B$. aphidicola APS showed that the network of this endosymbiont is also highly non-redundant, with $84 \%$ (94\% according to MOMA) of genes being essential (Thomas et al., 2009). Although differences in the estimation algorithm and the selected criteria make these numbers not directly comparable, it does come to show that the Buchnera lineage evolved nearly minimal networks before the divergence of the Aphidinae and Lachninae aphid subfamilies, about 90 Mya. Moreover, although there are no available metabolic reconstructions for others $S$. symbiotica strains, the essentiality in the metabolic network of S. symbiotica SCc probably represents the high degree of genome reduction occurring in its symbiotic lineage, at $1.76 \mathrm{Mb}$ and only $672 \mathrm{CDS}$ (Lamelas et al., 2011b). This is likely the result of more recent evolutionary processes, since S. symbiotica from hosts within the Aphidinae subfamily display genomes larger than $2.5 \mathrm{Mb}$ and contain over $2000 \mathrm{CDSs}$ (Burke and Moran, 2011; Foray et al., 2014; Manzano-Marín and
Latorre, 2016). This might indicate that the genome reduction process in $S$. symbiotica SCc occurred after the divergence of the Lachninae subfamily, ca. 55 Mya. Two other genomes from obligate $S$. symbiotica strains have been described within this subfamily, obtained from Tuberolachnus salignus (tribe Tuberolachnini) and Cinara tujafilina (Eulachnini). The former contains a genome of only $650 \mathrm{~kb}$ and 495 CDSs (ManzanoMarín et al., 2016), while the genome of the latter is in an early stage of reduction at $2.5 \mathrm{Mb}$ and $1602 \mathrm{CDSs}$ (ManzanoMarín and Latorre, 2014). If the transmission of Serratia was vertical and no replacement occurred in the C. tujafilina clade, as it has been suggested (Manzano-Marín and Latorre, 2016), extreme reductive processes observed in the iSCc236 network may have been even more recent, possibly occurring no longer than 40 Mya.

Altogether, the two metabolic networks involved in the $C$. cedri consortium are highly constrained and fragile. This is also shown in the list of metabolic requirements that these organisms exhibit, which is increased by the high number of full and partial pathways that have been lost in both members of the consortium. Moreover, they show a high degree of integration, where both members have suffered massive losses, presumably due to division of labor with the bacterial partner. Cases of such losses are, for instance, the B. aphidicola BCc loss of the ability to produce cofactors like siroheme, biotin or THF, and, in $S$. symbiotica SCc, of the ability to produce several amino acids such as phenylalanine, threonine and branched amino acids. The model $i$ BSCc establishes three cases of pathway sharing between the two symbionts, namely the biosynthesis of tryptophan, biotin and THF. These three pathways are partitioned between the two bacteria at the level of specific metabolites: anthranilate, $8 \mathrm{AONN}$ and shikimate, respectively (see Figure 2 ). Those three exchanged metabolites are among the most permeable ones of the participant intermediates, as it was predicted by our previous chemoinformatic analysis on metabolic complementation (Mori et al., 2016). Finally, the endosymbiont metabolic networks also show the need for metabolic complementation from compounds synthesized by the host. This is reflected by the requirement for the incorporation of metabolic intermediaries, not just endproducts, from the external compartment. Simulations with the metabolic network of S. symbiotica SCc show that two biosynthetic pathways are completed by the host. The loss of the first activities in the biosynthesis of siroheme and coenzyme A generate the need for the import of metabolic intermediaries. A similar case has been previously observed in Blattabacterium, symbiont of the cockroach Blattella germanica, where the initial steps in the biosynthesis of terpenes have been lost (Ponce-deLeon et al., 2013). These events are likely to commonly evolve in organisms under genome reduction processes, enabled by key factors such as the redundancy of pathways and the feasibility for transport due to permeability of the compound or to the existence or exaptation of generalist transporters.

\section{Emergence of Metabolic Complementation in Endosymbiotic Consortia}

The simulations performed in this study with the reconstructed model of the consortium, $i$ BSCc, reproduced the cases of metabolic complementation between the two bacterial partners 
that have been described in the literature, and predicted an additional one, the one involving the synthesis of THF in $S$. symbiotica SCc from the shikimate provided by B. aphidicola $\mathrm{BCc}$. The emergence of metabolic complementation is a complex phenomenon and is not well understood from a theoretical perspective. Tryptophan biosynthesis is highly regulated at the transcriptional, translational and posttranslational levels. Notably, this pathway is negatively regulated through attenuation of the transcription of anthranilate synthase by tryptophan (Crawford, 1989), a process that has been suggested to facilitate the emergence of metabolic complementation (Mori et al., 2016). Genes for tryptophan synthesis from chorismate are present in most of the sequenced B. aphidicola genomes. In the cedar aphid, the genes encoding this pathway are divided in a $B$. aphidicola BCc plasmid containing $\operatorname{trpEG}$, and a $S$. symbiotica SCc operon containing trpABCD. A strikingly identical case has been found to have occurred convergently in separate lineages of Buchnera and Serratia in the aphid T. salignus (Manzano-Marín et al., 2016). Moreover, the same complementation has been described in a completely different system, the one formed by ca. Carsonella ruddii and secondary symbionts related to Sodalis and Moranella, in psyllids (Sloan and Moran, 2012). More complex cases of complementation in tryptophan biosynthesis also exist, such as the case of the mealybug symbionts, where this pathway seems to require the transport of multiple intermediaries, possibly including anthranilate (McCutcheon and von Dohlen, 2011).

Under this perspective, our simulations of all alternative GLRPs of metabolic complementations applied to the three shared pathways yielded novel results. We constructed models representing alternative GLRPs from a hypothetical ancestor containing intact pathways in both symbionts, and compared how well they performed in maximizing the production of tryptophan, THF, biotin and meta-biomass, assuming that BCc and SCc are in proportion 1:1 in all the explored scenarios. Surprisingly, we found that, in the case of the tryptophan, the GLRP exhibited in the cedar aphid consortium behaved nearly optimal, and represented a quasi-minimal design in doing so. These results show that, from a structural point of view, the actual distribution or division of a metabolic pathway between two organisms can perform almost as well as their ancestor while using a smaller gene repertoire. Although the cost of protein synthesis and genome replication cannot be easily integrated in this kind of analysis, it is expected that a reduction in such costs will improve the growth efficiency of an organism (Mori et al., 2016). On the other hand, the results indicate that the GLRP exhibited in the biotin and THF pathways is suboptimal, and there are others GLRPs that allow a greater flux using the same number of genes. However, it is worth noting that the demand for these cofactors is probably much smaller than the demand for tryptophan, and thus the selective pressure for an efficient production of THF and biotin may be less stringent than the case of the amino acid. This situation may also reflect the limits of the simultaneous optimization of the diverse metabolic performances of a given network. In any case, the GLRP of the tryptophan biosynthesis exhibited by the consortium of the cedar aphid is convergent with the metabolic solution observed in the symbiotic consortium of the psyllid Heteropsylla cubana (Sloan and Moran, 2012).

On the other hand, the reduction experiment performed evaluating the meta-biomass equation indicate that the global GLRP exhibited by the cedar aphid consortium, is quasi-optimal in terms of yield, and nearly minimal in terms of gene number. Moreover, additional factors can influence the given structure, and confer more benefits. For instance, the fact that the first and the last steps of the pathway are performed in different compartments reduces the possibility that an accumulation of the end-product tryptophan would inhibit the first biosynthetic step by attenuation. This is despite the fact that the inhibition binding site for tryptophan is highly conserved (Mori et al., 2016), which might be due to constraints in the enzyme functional architecture. Besides the structure of the complementation, the pathway kinetics is also likely to be involved in the function and evolution of the metabolic complementation. However, the complexity associated to a kinetic model and the lack of experimentally-based parameters makes such a model implausible at a genome scale as of today. Future efforts to model complete metabolic systems at a genome scale beyond stoichiometric constraints, by adding reaction kinetics and higher-level processes, such as the cost of protein production and turnover, will shed more light into the structure, function and evolution of metabolism, and in the emergence of metabolic complementation.

\section{CONCLUSIONS}

The results and predictions obtained from GEMs, besides their intrinsic values, are useful as a tool to refine genomic and metabolic annotations. They also establish a powerful framework to interpret complex patterns of co-evolution, such as metabolic complementation. Here, we have reconstructed two genome-scale models from highly genome-reduced bacterial endosymbionts and integrated these models into a consortium model. Using this model we were able to study: (1) the requirements and exports of the bacterial partners to the host and to each other; (2) the robustness associated to reduced metabolic networks individually and by co-integration; and (3) the evolutionary constraints in the emergence of metabolic complementation designs. We could corroborate previously suggested scenarios for metabolic capabilities based on comparative genomic analyses. We also established that the cedar aphid consortium is composed not only of individual highly-reduced symbionts, but also that it is not far from a complete loss of metabolic redundancy and flexibility, thus making it a highly fragile partnership. Finally, we also showed that the patterns of metabolic complementation in this consortium are nearly minimal, in terms of gene content, and exhibit an almost optimal growth rate, and tryptophan production, with respect to a putative ancestor where the complemented pathways are still completely encoded by each symbiont. Therefore, our results suggest a higher role of adaptive evolution in the emergence of metabolic complementation than 
previously thought, and more studies in different consortia with both similar and different patterns of complementation designs will be invaluable to confirm the generality of these conclusions.

\section{AUTHOR CONTRIBUTIONS}

MP-d-L, FM, and JP conceived the work. MP-d-L, DT, and JC-E developed the models. MP-d-L performed the simulations and analyzed the data. All the authors discussed the results and wrote the manuscript.

\section{ACKNOWLEDGMENTS}

We would like to thank the Obra Social Programme of La Caixa Savings Bank for the doctoral fellowship granted to JC-E. Financial support from Spanish Government (grant reference: BFU2015-64322-C2-1-R co-financed by FEDER funds and Ministerio de Economía y Competitividad) and Generalitat Valenciana (grant reference: PROMETEOII/2014/065) is gratefully acknowledged. MM acknowledges financial support from Sapienza, University of Rome. DT acknowledges support by a European Union grant from the Marie Curie ITN SYMBIOMICS (264774) and a grant from the Knut and Alice Wallenberg Foundation (2012.0075), given to Björn Andersson (Karolinska Institute) and Siv Andersson (Uppsala University).

\section{SUPPLEMENTARY MATERIAL}

The Supplementary Material for this article can be found online at: https://www.frontiersin.org/articles/10.3389/fmicb. 2017.02290/full\#supplementary-material

Supplementary Text S1 | Extended results. The text includes four sections, two including details about the reconstruction and metabolic capabilities of the endosymbiont individual models. The other two sections correspond to the estimations of the proteome composition of $C$. cedri and to the construction of the meta-biomass.

Supplementary Text S2 | Extended Materials and Methods. The text includes details on the annotated genomes, constraint-based methods employed, as well as the reconstruction of the plausible ancestor model of $C$. cedri used as a reference point in the reduction experiments.

Supplementary Figure S1 | Conversion map predicted by iBCc98 and iSCc236. Rows represent imported compounds, and columns, biomass components synthesized by each organism. Colored squares in the same columns indicate the set of compounds required for the biosynthesis of a component. Purple and blue squares correspond to capabilities predicted by iBCc98 and iSCc236, respectively. Marked columns $(*)$ correspond to compounds produced by both bacteria

Supplementary Figure S2 | Optimal production rates of tryptophan for the reduced gene loss and retention experiments. Normalized optimal production rate of tryptophan with respect to the optimal value exhibited. The small arrow denotes the case of iBSCc, i.e., the cedar aphid consortium.

Supplementary Figure S3 | Optimal production rates of tetrahydrofolate for the reduced gene loss and retention experiments. Normalized optimal production rate of tetrahydrofolate with respect to the optimal value exhibited. The small arrow denote the case of $i \mathrm{BSC}$, i.e., the cedar aphid consortium.

Supplementary Figure S4 | Optimal production rates of biotin for the reduced gene loss and retention experiments. Normalized optimal production rate of biotin with respect to the optimal value exhibited. The small arrow denote the case of iBSCc, i.e., the cedar aphid consortium.

Supplementary Table S1 | Genome-scale metabolic model of Serratia symbiotica SCc iSCc236. The spreadsheet in XLS format includes the iSCc236 model description. It contains three tables: one that corresponds to the reactions; the second includes exchange fluxes; and in the third is the information of the metabolites

Supplementary Table S2 | Genome-scale metabolic model of Buchnera aphidicola BCc iBCc98. The file corresponds to the representation of the iBCc98 model in spreadsheet format, and includes the following four sheets: (i) the list of reactions; (ii) listing of exchange fluxes; (iii) listing of metabolites; (iv) the list of reactions and metabolic genes that were not included in the model.

Supplementary Table S3 | Genome-scale metabolic model of endosymbiotic consortium of the cedar aphid C. cedri iBSCc. The file corresponds to the representation of the $i \mathrm{BSC}$ model in spreadsheet format, and includes the following fiver sheets: (i) the list of reactions; (ii) listing of exchange fluxes; (iii) listing of metabolites; (iv) the optimal flux distribution that maximizes the growth of the whole system; and (v) the exchange patterns between the consortium members.

Supplementary Table S4 | Results from the robustness analysis. The table include two sheets. The first sheet lists the results from the single $\mathrm{KO}$ in-silico experiments, whereas the second sheet includes the results of the double KO in-silico experiments.

Supplementary Table S5 | Results from in-silico reduction experiments. The table includes two sheets. The first sheet summarizes the results from the reduction experiments and the second sheet includes a table with a mapping between Enzyme Subsets and the reactions IDs.

Supplementary Table S6 | Results from the reduced in-silico reduction experiments. The table includes three sheets. Each sheet includes the results of the reduction experiments for the tryptophan, tetrahydrofolate and biotin biosynthesis, when only the genes involved in each single pathway are considered for the experiment.

Supplementary File S1 | SBML models: iBCc98, iSCc236, and iBSCc.

\section{REFERENCES}

Altschul, S. F., Gish, W., Miller, W., Myers, E. W., and Lipman, D. J. (1990). Basic local alignment search tool. J. Mol. Biol. 215, 403-410. doi: 10.1016/S0022-2836(05)80360-2

Ankrah, N. Y. D., Luan, J., and Douglas, A. E. (2017). Cooperative metabolism in a three-partner insect-bacterial symbiosis revealed by metabolic modeling. J. Bacteriol. 199:e00872-16. doi: 10.1128/JB.00 872-16

Baumann, P., Moran, N. A., and Baumann, L. (2006). "Bacteriocyte-associated endosymbionts of insects," in The Prokaryotes, Vol. 1, eds M. Dworkin, S. Falkow, E. Rosenberg, and K.-H. Stackebrandt (New York, NY: Springer), 403-438.

Belda, E., Silva, F. J., Peretó, J., and Moya, A. (2012). Metabolic networks of Sodalis glossinidius: a systems biology approach to reductive evolution. PLoS ONE 7:e30652. doi: 10.1371/journal.pone.0030652

Burgard, A. P., Vaidyaraman, S., and Maranas, C. D. (2001). Minimal reaction sets for Escherichia coli metabolism under different growth requirements and uptake environments. Biotechnol. Prog. 17, 791-797. doi: 10.1021/bp0100880

Burke, G. R., and Moran, N. A. (2011). Massive genomic decay in Serratia symbiotica, a recently evolved symbiont of aphids. Genome Biol. Evol. 3, 195-208. doi: 10.1093/gbe/evr002

Calle-Espinosa, J., Ponce-de-Leon, M., Santos-Garcia, D., Silva, F. J., Montero, F., and Peretó, J. (2016). Nature lessons: the whitefly bacterial endosymbiont is a minimal amino acid factory with unusual energetics. J. Theor. Biol. 407, 303-317. doi: 10.1016/j.jtbi.2016.07.024 
Caspi, R., Altman, T., Billington, R., Dreher, K., Foerster, H., Fulcher, C. A., et al. (2014). The MetaCyc database of metabolic pathways and enzymes and the BioCyc collection of Pathway/Genome Databases. Nucleic Acids Res. 42, D459-D471. doi: 10.1093/nar/gkt1103

Charles, H., Balmand, S., Lamelas, A., Cottret, L., Pérez-Brocal, V., Burdin, B., et al. (2011). A genomic reappraisal of symbiotic function in the aphid/Buchnera symbiosis: reduced transporter sets and variable membrane organisations. PLoS ONE 6:e29096. doi: 10.1371/journal.pone.0029096

Crawford, I. P. (1989). Evolution of a biosynthetic pathway: the tryptophan paradigm. Annu. Rev. Microbiol. 43, 567-600. doi: 10.1146/annurev.mi.43. 100189.003031

Douglas, A. E., Minto, L. B., and Wilkinson, T. L. (2001). Quantifying nutrient prosduction by the microbial symbionts in an aphid. J. Exp. Biol. 204, 349-358.

Ebrahim, A., Lerman, J. A., Palsson, B. O., Hyduke, D. R., and Ebrahim, A., Lerman, J. A., et al. H. D. (2013). COBRApy: COnstraints-based reconstruction and analysis for python. BMC Syst. Biol. 7:74. doi: 10.1186/1752-0509-7-74

Faust, K., and Raes, J. (2012). Microbial interactions: from networks to models. Nat. Rev. Microbiol. 10, 538-550. doi: 10.1038/nrmicro2832

Foray, V., Grigorescu, A. S., Sabri, A., Haubruge, E., Lognay, G., Francis, F., et al. (2014). Whole-genome sequence of Serratia symbiotica Strain CWBI-2.3T, a free-living symbiont of the black bean aphid aphis fabae. Genome Announc. 2:e00767-14. doi: 10.1128/genomeA.00767-14

Germerodt, S., Bohl, K., Lück, A., Pande, S., Schröter, A., Kaleta, C., et al. (2016). Pervasive selection for cooperative cross-feeding in bacterial communities. PLoS Comput. Biol. 12:e1004986. doi: 10.1371/journal.pcbi.1004986

Gil, R., and Peretó, J. (2015). Small genomes and the difficulty to define minimal translation and metabolic machineries. Front. Ecol. Evol. 3:123. doi: 10.3389/fevo.2015.00123

Gomez-Valero, L., Soriano-Navarro, M., Perez-Brocal, V., Heddi, A., Moya, A., Garcia-Verdugo, J. M., et al. (2004). Coexistence of Wolbachia with Buchnera aphidicola and a Secondary Symbiont in the Aphid Cinara cedri. J. Bacteriol. 186, 6626-6633. doi: 10.1128/JB.186.19.6626-6633.2004

González-Domenech, C. M., Belda, E., Patiño-Navarrete, R., Moya, A., Peretó, J., and Latorre, A. (2012). Metabolic stasis in an ancient symbiosis: genomescale metabolic networks from two Blattabacterium cuenoti strains, primary endosymbionts of cockroaches. BMC Microbiol. 12 (Suppl. 1):S5. doi: 10.1186/ 1471-2180-12-S1-S5

Gosalbes, M. J., Lamelas, A., Moya, A., and Latorre, A. (2008). The striking case of tryptophan provision in the cedar aphid Cinara cedri. J. Bacteriol. 190, 6026-6029. doi: 10.1128/JB.00525-08

Graber, J. R., and Breznak, J. A. (2005). Folate cross-feeding supports symbiotic homoacetogenic spirochetes. Appl. Environ. Microbiol. 71, 1883-1889. doi: 10.1128/AEM.71.4.1883-1889.2005

Großkopf, T., and Soyer, O. S. (2016). Microbial diversity arising from thermodynamic constraints. ISME J. 10, 2725-2733. doi: 10.1038/ismej.2016.49

Hansen, A. K., and Moran, N. A. (2011). Aphid genome expression reveals hostsymbiont cooperation in the production of amino acids. Proc. Natl. Acad. Sci. U.S.A. 108, 2849-2854. doi: 10.1073/pnas.1013465108

Henry, C. S., DeJongh, M., Best, A. A., Frybarger, P. M., Linsay, B., and Stevens, R. L. (2010). High-throughput generation, optimization and analysis of genome-scale metabolic models. Nat. Biotechnol. 28, 977-982. doi: 10.1038/ nbt. 1672

Karp, P. D., Latendresse, M., Paley, S. M., Ong, M. K. Q., Billington, R., Kothari, A., et al. (2015). Pathway tools version 19.0: integrated software for pathway/genome informatics and systems biology. Brief. Bioinform. 17, 877-890. doi: 10.1093/bib/bbv079

Kerner, A., Park, J., Williams, A., and Lin, X. N. (2012). A programmable Escherichia coli consortium via tunable symbiosis. PLOS ONE 7:e34032. doi: 10.1371/journal.pone.0034032

Lamelas, A., Gosalbes, M. J., Manzano-Marín, A., Peretó, J., Moya, A., and Latorre, A. (2011a). Serratia symbiotica from the aphid Cinara cedri: a missing link from facultative to obligate insect endosymbiont. PLoS Genet. 7:e1002357. doi: 10.1371/journal.pgen.1002357

Lamelas, A., Gosalbes, M. J., Moya, A., and Latorre, A. (2011b). New clues about the evolutionary history of metabolic losses in bacterial endosymbionts, provided by the genome of Buchnera aphidicola from the aphid Cinara tujafilina. Appl. Environ. Microbiol. 77, 4446-4454. doi: 10.1128/AEM.00141-11
Li, L., Stoeckert, C. J., and Roos, D. S. (2003). OrthoMCL: identification of ortholog groups for eukaryotic genomes. Genome Res. 13, 2178-2189. doi: $10.1101 /$ gr.1224503

López-Sánchez, M. J., Neef, A., Peretó, J., Patiño-Navarrete, R., Pignatelli, M., Latorre, A., et al. (2009). Evolutionary convergence and nitrogen metabolism in Blattabacterium strain Bge, primary endosymbiont of the cockroach Blattella germanica. PLoS Genet. 5:e1000721. doi: 10.1371/journal.pgen.1000721

Macdonald, S. J., Lin, G. G., Russell, C. W., Thomas, G. H., and Douglas, A. E. (2012). The central role of the host cell in symbiotic nitrogen metabolism. Proc. Biol. Sci. 279, 2965-2973. doi: 10.1098/rspb.2012.0414

MacDonald, S. J., Thomas, G. H., and Douglas, A. E. (2011). Genetic and metabolic determinants of nutritional phenotype in an insect-bacterial symbiosis. Mol. Ecol. 20, 2073-2084. doi: 10.1111/j.1365-294X.2011.05031.x

Manzano-Marín, A., and Latorre, A. (2014). Settling down: the genome of Serratia symbiotica from the aphid Cinara tujafilina zooms in on the process of accommodation to a cooperative intracellular life. Genome Biol. Evol. 6, 1683-1698. doi: 10.1093/gbe/evu133

Manzano-Marín, A., and Latorre, A. (2016). Snapshots of a shrinking partner: genome reduction in Serratia symbiotica. Sci. Rep. 6:32590. doi: $10.1038 /$ srep32590

Manzano-Marín, A., Simon, J. C., and Latorre, A. (2016). Reinventing the wheel and making it round again: evolutionary convergence in Buchneraserratia symbiotic consortia between the distantly related Lachninae aphids Tuberolachnus salignus and Cinara cedri. Genome Biol. Evol. 8, 1440-1458. doi: 10.1093/gbe/evw085

Martínez-Cano, D. J., Reyes-Prieto, M., Martínez-Romero, E., Partida-Martínez, L. P., Latorre, A., Moya, A., et al. (2015). Evolution of small prokaryotic genomes. Front. Microbiol. 5:742. doi: 10.3389/fmicb.2014.00742

McCutcheon, J. P. (2016). From microbiology to cell biology: when an intracellular bacterium becomes part of its host cell. Curr. Opin. Cell Biol. 41, 132-136. doi: $10.1016 /$ j.ceb.2016.05.008

McCutcheon, J. P., and Moran, N. A. (2011). Extreme genome reduction in symbiotic bacteria. Nat. Rev. Microbiol. 10, 13-26. doi: 10.1038/nrmicro2670

McCutcheon, J. P., and von Dohlen, C. D. (2011). An interdependent metabolic patchwork in the nested symbiosis of mealybugs. Curr. Biol. 21, 1366-1372. doi: 10.1016/j.cub.2011.06.051

Moran, N. A. (1996). Accelerated Evolution and Muller's Rachet in Endosymbiotic Bacteria. Proc. Natl. Acad. Sci. U.S.A. 93, 2873-2878. doi: $10.1073 /$ pnas.93.7.2873

Moran, N. A., and Bennett, G. M. (2014). The tiniest tiny genomes. Annu. Rev. Microbiol. 68, 195-215. doi: 10.1146/annurev-micro-091213-112901

Mori, M., Ponce-de-León, M., Peret,ó, J., and Montero, F. (2016). Metabolic complementation in bacterial communities: necessary conditions and optimality. Front. Microbiol. 7:1553. doi: 10.3389/fmicb.2016.01553

Moya, A., Peretó, J., Gil, R., and Latorre, A. (2008). Learning how to live together: genomic insights into prokaryote-animal symbioses. Nat. Rev. Genet. 9, 218-229. doi: 10.1038/nrg2319

Orth, J. D., Conrad, T. M., Na, J., Lerman, J. A., Nam, H., Feist, A. M., et al. (2011). A comprehensive genome-scale reconstruction of Escherichia coli metabolism-2011. Mol. Syst. Biol. 7:535. doi: 10.1038/msb.2011.65

Orth, J. D., Thiele, I., and Palsson, B. Ø. (2010). What is flux balance analysis? Nat. Biotechnol. 28, 245-248. doi: 10.1038/nbt.1614

Pál, C., Papp, B., Lercher, M. J., Csermely, P., Oliver, S. G., and Hurst, L. D. (2006). Chance and necessity in the evolution of minimal metabolic networks. Nature 440, 667-670. doi: 10.1038/nature04568

Patiño-Navarrete, R., Piulachs, M.-D., Belles, X., Moya, A., Latorre, A., and Peretó, J. (2014). The cockroach Blattella germanica obtains nitrogen from uric acid through a metabolic pathway shared with its bacterial endosymbiont. Biol. Lett. 10:20140407. doi: 10.1098/rsbl.2014.0407

Pérez-Brocal, V., Gil, R., Ramos, S., Lamelas, A., Postigo, M., Michelena, J. M., et al. (2006). A small microbial genome: the end of a long symbiotic relationship? Science 314, 312-313. doi: 10.1126/science.1130441

Poliakov, A., Russell, C. W., Ponnala, L., Hoops, H. J., Sun, Q., Douglas, A. E., et al. (2011). Large-scale label-free quantitative proteomics of the pea aphid-Buchnera symbiosis. Mol. Cell. Proteomics 10:M110.007039. doi: 10.1074/mcp.M110.007039

Ponce-de-Leon, M., Montero, F., and Peretó, J. (2013). Solving gap metabolites and blocked reactions in genome-scale models: application to the 
metabolic network of Blattabacterium cuenoti. BMC Syst. Biol. 7:114. doi: 10.1186/1752-0509-7-114

Price, D. R. G., and Wilson, A. C. C. (2014). A substrate ambiguous enzyme facilitates genome reduction in an intracellular symbiont. BMC Biol. 12:110. doi: 10.1186/s12915-014-0110-4

Reed, J. L., Vo, T. D., Schilling, C. H., and Palsson, B. O. (2003). An expanded genome-scale model of Escherichia coli K-12 \{(iJR904\} \{GSM/GPR)\}. Genome Biol. 4:R54. doi: 10.1186/gb-2003-4-9-r54

Richards, S., Gibbs, R. A., Gerardo, N. M., Moran, N., Nakabachi, A., Stern, D., et al. (2010). Genome sequence of the pea aphid Acyrthosiphon pisum. PLoS Biol. 8:e1000313. doi: 10.1371/journal.pbio.1000313

Russell, C. W., Bouvaine, S., Newell, P. D., and Douglas, A. E. (2013). Shared metabolic pathways in a coevolved insect-bacterial symbiosis. Appl. Environ. Microbiol. 79, 6117-6123. doi: 10.1128/AEM.01543-13

Russell, C. W., Poliakov, A., Haribal, M., Jander, G., van Wijk, K. J., and Douglas, A. E. (2014). Matching the supply of bacterial nutrients to the nutritional demand of the animal host. Proc. Biol. Sci. 281:20141163. doi: 10.1098/rspb.2014.1163

Sabater-Muñoz, B., Toft, C., Alvarez-Ponce, D., and Fares, M. A. (2017). Chance and necessity in the genome evolution of endosymbiotic bacteria of insects. ISME J. 11, 1291-1304. doi: 10.1038/ismej.2017.18

Schellenberger, J., Park, J. O., Conrad, T. M., and Palsson, B. Ø. (2010). BiGG: a Biochemical Genetic and Genomic knowledgebase of large scale metabolic reconstructions. BMC Bioinformatics 11:213. doi: 10.1186/1471-2105-11-213

Segrè, D., Vitkup, D., and Church, G. M. (2002). Analysis of optimality in natural and perturbed metabolic networks. Proc. Natl. Acad. Sci. U.S.A. 99, 15112-15117. doi: 10.1073/pnas.232349399

Seth, E. C., and Taga, M. E. (2014). Nutrient cross-feeding in the microbial world. Front. Microbiol. 5:350. doi: 10.3389/fmicb.2014.00350

Sloan, D. B., and Moran, N. A. (2012). Genome reduction and co-evolution between the primary and secondary bacterial symbionts of psyllids. Mol. Biol. Evol. 29, 3781-3792. doi: 10.1093/molbev/mss 180

Thiele, I., and Palsson, B. Ø. (2010). A protocol for generating a highquality genome-scale metabolic reconstruction. Nat. Protoc. 5, 93-121. doi: 10.1038/nprot.2009.203

Thomas, G. H., Zucker, J., Macdonald, S. J., Sorokin, A., Goryanin, I., and Douglas, A. E. (2009). A fragile metabolic network adapted for cooperation in the symbiotic bacterium Buchnera aphidicola. BMC Syst. Biol. 3:24. doi: 10.1186/1752-0509-3-24

Van Leuven, J. T., Meister, R. C., Simon, C., and McCutcheon, J. P. (2014) Sympatric speciation in a bacterial endosymbiont results in two genomes with the functionality of one. Cell 158, 1270-1280. doi: 10.1016/j.cell.2014. 07.047

Vellozo, A. F., Véron, A. S., Baa-Puyoulet, P., Huerta-Cepas, J., Cottret, L., Febvay, G., et al. (2011). CycADS: an annotation database system to ease the development and update of BioCyc databases. Database 2011:bar008. doi: 10.1093/database/bar008

Wagner, A. (2005). Distributed robustness versus redundancy as causes of mutational robustness. Bioessays 27, 176-188. doi: 10.1002/bies.20170

Wernegreen, J. J. (2002). Genome evolution in bacterial endosymbionts of insects. Nat. Rev. Genet. 3, 850-861. doi: 10.1038/nrg931

Whitehead, L. F., and Douglas, A. E. (1993). Populations of Symbiotic Bacteria in the Parthenogenetic Pea Aphid (Acyrthosiphon pisum) Symbiosis. Proc. R. Soc. B Biol. Sci. 254, 29-32. doi: 10.1098/rspb.1993.0122

Wu, D., Daugherty, S. C., Van Aken, S. E., Pai, G. H., Watkins, K. L., Khouri, H., et al. (2006). Metabolic complementarity and genomics of the dual bacterial symbiosis of sharpshooters. PLoS Biol. 4:e188 doi: 10.1371/journal.pbio.0040188

Zientz, E., Dandekar, T., and Gross, R. (2004). Metabolic interdependence of obligate intracellular bacteria and their insect hosts. Microbiol. Mol. Biol. Rev. 68, 745-770. doi: 10.1128/MMBR.68.4.745-770.2004

Conflict of Interest Statement: The authors declare that the research was conducted in the absence of any commercial or financial relationships that could be construed as a potential conflict of interest.

Copyright (C) 2017 Ponce-de-Leon, Tamarit, Calle-Espinosa, Mori, Latorre, Montero and Pereto. This is an open-access article distributed under the terms of the Creative Commons Attribution License (CC BY). The use, distribution or reproduction in other forums is permitted, provided the original author(s) or licensor are credited and that the original publication in this journal is cited, in accordance with accepted academic practice. No use, distribution or reproduction is permitted which does not comply with these terms. 